\title{
A New Approach in the Simplification of a Multiple-Beam Forming Network Based on CORPS Using Compressive Arrays
}

\author{
Armando Arce, ${ }^{1}$ Leonardo F. Yepes, ${ }^{1}$ David H. Covarrubias, ${ }^{1}$ and Marco A. Panduro ${ }^{2}$ \\ ${ }^{1}$ Department of Electronics and Telecommunications, CICESE Research Centre, 22860 Ensenada, Mexico \\ ${ }^{2}$ Unidad Academica Multidisciplinaria Reynosa-Rhode, Universidad Autonoma de Tamaulipas, 88779 Reynosa, Mexico
}

Correspondence should be addressed to Armando Arce, arce@cicese.edu.mx

Received 14 March 2012; Accepted 23 July 2012

Academic Editor: Carlos del Rio Bocio

Copyright ( $) 2012$ Armando Arce et al. This is an open access article distributed under the Creative Commons Attribution License, which permits unrestricted use, distribution, and reproduction in any medium, provided the original work is properly cited.

\begin{abstract}
This research paper deals with a innovative way to simplify the design of beam-forming networks (BFNs) for multibeam steerable antenna arrays based on coherently radiating periodic structures (CORPS) technology using the noniterative matrix pencil method (MPM). This design approach is based on the application of the MPM to linear arrays fed by CORPS-BFN configurations to further reduce the complexity of the beam-forming network. Two 2-beam design configurations of CORPS-BFN for a steerable linear array are analyzed and compared using this compressive method. Simulation results show the effectiveness and advantages of applying the MPM on BFNs based on CORPS exploiting the nonuniformity of the antenna elements. Furthermore, final results show that the integration of CORPS-BFN and MPM reduces the entire antenna system including the antenna array and the beam-forming network subsystem resulting in a substantial simplification in such systems.
\end{abstract}

\section{Introduction}

The increase of technologies that require advanced features in modern antenna systems such as the dynamic redirection and reorganization of its services combined with the ability to handle multiple independent beams, require solutions that combine these characteristics efficiently. This is the case of last-generation multiple-beam forming networks (MBFN), which combine properties of phased arrays with BFNs to generate multiple independent beams that can be also directed to different directions of interest. In addition, an open research area in modern antenna systems is associated with reducing or simplifying the beam forming network including the number of antenna elements, for the purpose of reducing weight, size, and cost of a complete antenna system.

This work presents the technological concept of coherently radiating periodic structures (CORPS) [1-5] for a linear antenna array applying a compression technique known as matrix pencil method (MPM) $[6,7]$ at the output of the feeding network to reduce the number of antenna elements.
Recent research work in CORPS-BFN is focused in using the feeding network for specific applications [2], the development of evolutions to improve efficiency [4], and the simplification of the network $[3,5]$. In this last research area, the results have shown a significant simplification of the BFN combined with an aperture reuse especially in the planar array geometry [3]. However, the study of new structures for designing CORPS-BFN can be focused on design cases where the simplification and performance of the network can be improved not only for the antenna geometry but by advanced analytical techniques for the compression of antenna arrays used in the synthesis of antennas.

This research work based on features of CORPS-BFN and the matrix pencil method introduces a novel way to analyze and reduce a feeding network for scannable multibeam linear antenna arrays. In this manner, the objective of this work is to demonstrate the possible simplification of a CORPS-BFN applying the MPM to the network to compress the antenna elements.

The main contribution of this paper is to illustrate a new perspective in the design simplification of CORPS-BFN considering scannable multi-beam linear arrays including 


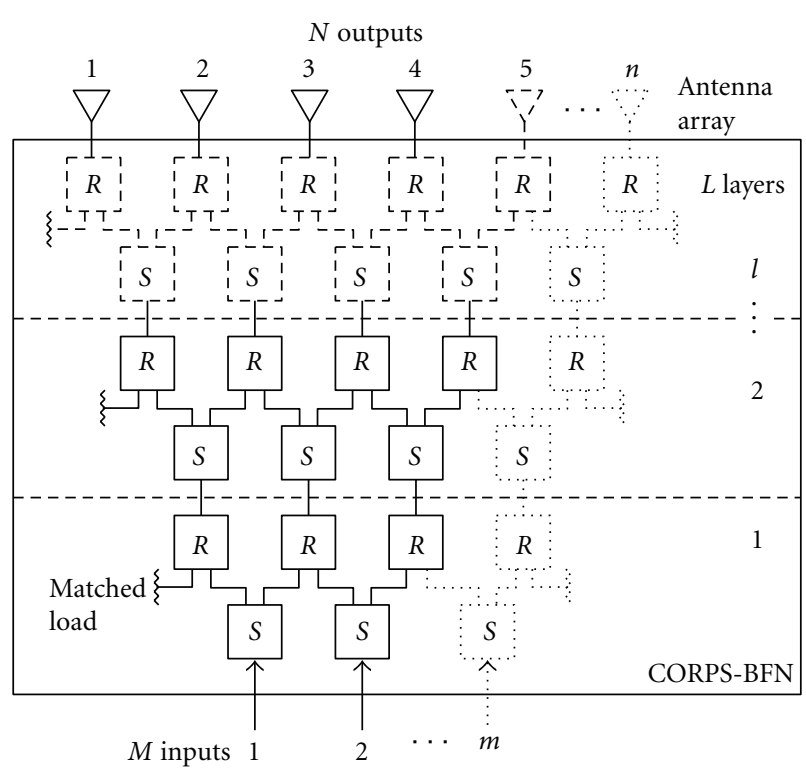

FIGURE 1: Schematic of CORPS beam-forming network with $S$ and $R$ nodes of $M$ inputs, $N$ outputs, and $L$ layers.

the compression of the antenna array based on a noniterative method called matrix pencil method.

The paper is organized as follows. Section 2 describes the behavior of a CORPS-BFN followed by a description of the optimization process applied and the mathematical formulation for the MPM. The simulation set up and results are presented in Section 3. Finally, conclusions of this work are presented in Section 4.

\section{Theoretical Study}

2.1. CORPS-BFN Model. The BFN addressed in this work is based on the fundamental principles of periodic structures called CORPS [1]. A CORPS-BFN is typically implemented by identical unit cells. These unit cells are used as basic nodes to form an entire feeding network, as previously discussed in [1-5]. In CORPS-BFN each node may act as a power combiner (Recombination $(R)$-node) or power splitter (Split $(S)$-node) by changing its relative position as shown in Figure 1.

A unit cell can be represented as a 3-port component characterized by the following scattering matrix [1]:

$$
[S]_{\text {cell }}=\frac{j}{\sqrt{2}}\left[\begin{array}{lll}
0 & 1 & 1 \\
1 & 0 & 0 \\
1 & 0 & 0
\end{array}\right] .
$$

Furthermore, in order to calculate the fields at the output of each unit cell $\left[V^{-}\right]_{\text {cell }}=\left[V_{1}^{-} V_{2}^{-} V_{3}^{-}\right]^{T}$ in (1) it can be used the next expression:

$$
\left[\begin{array}{l}
V_{1}^{-} \\
V_{2}^{-} \\
V_{3}^{-}
\end{array}\right]_{\text {cell }}=\left[\begin{array}{lll}
S_{11} & S_{12} & S_{13} \\
S_{21} & S_{22} & S_{23} \\
S_{31} & S_{32} & S_{33}
\end{array}\right]_{\text {cell }} \cdot\left[\begin{array}{c}
V_{1}^{+} \\
V_{2}^{+} \\
V_{3}^{+}
\end{array}\right]_{\text {cell }},
$$

where $[S]_{\text {cell }}=\left[S_{11} \cdots S_{33}\right]^{T}$ is the scattering matrix of a unit cell and $\left[V^{+}\right]_{\text {cell }}=\left[V_{1}^{+} V_{2}^{+} V_{3}^{+}\right]^{T}$ is the complex excitation (amplitude and phase) at the input ports of a unit cell.

Additionally, if we consider a linear array of $N$ antenna elements (one dimension). Its array factor for a set of complex inputs [a] feeding the CORPS-BFN is given by the next equation $[8]$ :

$$
\operatorname{AF}(\theta, \mathbf{a})=\sum_{n=1}^{N} a_{n} \exp \left[j k\left(d_{n} \cos \theta\right)\right]
$$

where $a_{n}$ represents the complex excitation of the $n$th antenna element of the array considering the the feeding network, $d_{n}$ indicates the position of the $n$th element of the array, and $\theta$ represents the angle of incidence of a plane wave.

The complex inputs feeding the CORPS network are included in the array factor considering the proposed inputs (i.e., the complex excitation to feed the $n$th radiator of the array) and a progressive phase excitation, given by

$$
\mathbf{a}=A_{i} \exp \left(j \xi_{i}\right) \exp \left(j \psi_{i}\right) \quad \text { for } 1 \leq i \leq M .
$$

The first term $A_{i} \exp \left(j \xi_{i}\right)$ in (4) denotes the typical complex excitation (amplitude and phase, resp.) where $\xi_{i} \in[0,180]$ and the second term $\exp \left(j \psi_{i}\right)$ adds a phase excitation in the complex inputs of the feeding network. Finally, $M$ represents the number of input ports of the network and the term $\psi_{i}$ is

$$
\psi_{i}=k\left[d m_{i} \cos \theta_{0}\right]
$$

In (5) $\left(\theta_{0}\right)$ indicates the direction of maximum radiation and the term $d m_{i}$ is given by

$$
d m_{i}=d_{i}+\left(\frac{M}{M-L}\right)
$$

where $M=N-1$ ( $N$ is the total number of antenna elements). More detailed information on the implementation of CORPS on linear arrays can be found in [2].

The optimization process followed to obtain nearoptimal solutions for the complex inputs to feed the BFN is described in the next subsection.

2.2. Optimization Process. The optimization process applied to the CORPS-BFN system is used to obtain complex excitations (amplitudes and phases) that should be directly applied at the input ports of the feeding network. In this paper, these excitations are determined by particle swarm optimization, a well-known metaheuristic in electromagnetic problems.

By analogy with the social behavior exhibited by biological communities to satisfy their immediate needs, in PSO the set of parameters to be optimized define a particle or potential solution. A collection of particles or individuals form the swarm (population) where each particle moves through one basic operator (velocity). In this way, the behavior of PSO can be summarized in the velocity operator $v_{k d}^{t}$ and a position equation $x_{k d}^{t}$, respectively [9], given by

$$
\begin{gathered}
v_{k d}^{t}=\omega v_{k d}^{t-1}+c_{1} \varepsilon_{1 k}\left(p_{k d}^{*}-x_{k d}^{t-1}\right) \\
+c_{2} \varepsilon_{2 k}\left(g_{d}^{*}-x_{k d}^{t-1}\right) \quad v_{k d}^{t} \leq v_{d, \max } \forall d, \\
x_{k d}^{t}=x_{k d}^{t-1}+v_{k d}^{t},
\end{gathered}
$$


where $\omega$ is the inertial weight, $c_{1}$ and $c_{2}$ are the acceleration constants. The terms represented by $\varepsilon$ are random numbers uniformly distributed in $U[0,1]$. The current personal best and global best are represented by $p_{k d}^{*}$ and $g_{d}^{*}$, respectively. Detailed information of the terminology can be found in [9].

The optimization process used by PSO follows the next steps: PSO generates particles as possible solutions. These particles or individuals are encoded in two vectors of real numbers which represent amplitudes and phase perturbations. Each particle generates an array factor of certain characteristics of side lobe level and directivity. Then the PSO operator of velocity (7) and the position equation (8) are used for moving each particle through the solution space. Finally, PSO finds a global solution that generates an array factor with minimum side lobe level and maximum directivity in the desired direction.

In this work, PSO is used just as an optimization tool for the advantages of the algorithm itself. The performance comparison between PSO and other algorithms for this purpose is out of the scope of this paper.

The objective function (Of) used can be written as

$$
\text { Of }=\min \left[\left(\frac{\left|\operatorname{AF}\left(\theta_{\mathrm{SLL}}, \mathbf{a}\right)\right|}{\max |\operatorname{AF}(\theta, \mathbf{a})|}\right)+\left(\frac{1}{D(\theta, \mathbf{a})}\right)\right],
$$

where $\left(\theta_{\mathrm{SLL}}\right)$ is the angle where the maximum side lobe is attained. The goal is to minimize the weighted sum that involves both objectives (side lobe level and directivity), which are uniformly weighted in the cost function.

The technique used in this work to reduce the number of antenna elements is described in the next subsection.

2.3. Matrix Pencil Method. In order to compress a onedimensional antenna array of $M$ elements using the matrix pencil method developed by Liu et al. [6], we can parameterize (3) in terms of $u$ (reference pattern) for sampling purposes, as follows:

$$
\mathrm{AF}_{\mathrm{ref}}(u)=\sum_{n=1}^{N} a_{n} \exp \left[j k\left(d_{n} u\right)\right]
$$

where $u=\cos \theta$, and must be resolved the following optimization problem:

$$
\begin{aligned}
& \min \{P\}, \\
& \text { Const. }\left\{\min _{\left\{I_{n}^{*}, d_{n}^{*}\right\}_{n=1, \ldots ., P}}\left\|\overline{\mathrm{AF}}_{\mathrm{ref}}(u)-\sum_{n=1}^{P} I_{n}^{*} e^{\left(j k d_{n}^{*} u\right)}\right\|_{L}\right\} \leq \xi,
\end{aligned}
$$

where $I_{n}^{*}$ and $d_{n}^{*}$ for all $n=1, \ldots, P$ are the complex excitation, and the position of the $P$ antenna elements obtained after the compression. The methodology for solving this problem consists of two stages, in the first one we compute the singular value decomposition (SVD) of the Hankel matrix $[\mathbf{Y}]$ formed by the $S$ samples of the parameter $u$ in the reference array factor $\overline{\mathrm{AF}}_{\text {ref }}\left(u_{s}\right)$. That is, for all $u_{s}=$ $s \Delta=s / S, s=-S, \ldots, 0, \ldots, S$ subject to the Nyquist sampling theorem condition $\Delta \leq \lambda / 2 d_{\max }$, where $d_{\max }=\max \left\{d_{n}\right\}$. In this way, we obtain the spectrum of the $E$ singular values which determines the elements to be discriminated in the calculation of the array factor according to the following expression:

$$
P=\min \left\{p ;\left|\frac{\sqrt{\sum_{n=p+1}^{E} \sigma_{n}^{2}}}{\sqrt{\sum_{n=1}^{p} \sigma_{n}^{2}}}\right|<\epsilon\right\} .
$$

The second step consists of using the MPM to estimate the parameters $\left\{z_{n}^{*}\right\}$ corresponding to the position of the new $P$ array elements. With the parameters $z_{n}^{*}$, it is possible to calculate the new position of the antenna elements $d_{n}$ as

$$
\begin{gathered}
d_{n}^{*}=\frac{1}{j k \Delta} \log \left(\hat{z}_{n}^{*}\right) ; \quad j=\sqrt{-1}, \\
I_{n}^{*}=\left([\hat{\mathbf{Z}}]^{H}[\hat{\mathbf{Z}}]\right)^{-1}[\hat{\mathbf{Z}}] \overline{\mathrm{AF}}_{\mathrm{ref}}\left(u_{s}\right),
\end{gathered}
$$

where

$$
\begin{gathered}
\hat{z}_{n}^{*}=\frac{z_{n}^{*}}{\left|z_{n}^{*}\right|}, \\
\overline{\mathrm{AF}}_{\mathrm{ref}}\left(u_{s}\right)=\left(\mathrm{AF}_{\mathrm{ref}}(-S), \mathrm{AF}_{\mathrm{ref}}(-S+1), \ldots, \mathrm{AF}_{\mathrm{ref}}(S)\right)^{T}, \\
{[\hat{\mathbf{z}}]=\left[\begin{array}{cccc}
\left(\hat{z}_{1}^{*}\right)^{-S} & \left(\hat{z}_{2}^{*}\right)^{-S} & \ldots & \left(\hat{z}_{P}^{*}\right)^{-S} \\
\left(\hat{z}_{1}^{*}\right)^{-S+1} & \left(\hat{z}_{2}^{*}\right)^{-S+1} & \ldots & \left(\hat{z}_{P}^{*}\right)^{-S+1} \\
\vdots & \vdots & \vdots & \vdots \\
\left(\hat{z}_{1}^{*}\right)^{S} & \left(\hat{z}_{2}^{*}\right)^{S} & \ldots & \left(\hat{z}_{P}^{*}\right)^{S}
\end{array}\right]_{(2 S+1) \times P} .}
\end{gathered}
$$

Detailed information on the methodology of the MPM and for the calculation of the parameters involved can be found in $[5,6]$.

\section{Simulation Setup and Results}

3.1. Case Study. To demonstrate the compression capabilities of the matrix pencil method together with CORPS technology applied to a linear antenna array, we proposed two configurations of 2-beam design for simplicity reasons, as follows:

(1) The first configuration (Figure 1) is a linear array system of 20 antenna elements with 19 feeding ports (i.e., $M=19$ and $N=20$ ) implemented as a CORPS$\mathrm{BFN}$ of one layer. We used 10 complex inputs to control 11 antennas (beam no. 1) and 9 complex inputs to control 10 antennas (beam no. 2). In this type of configuration based on subgroups of input ports, an orthogonal beam could be conformed and controlled by the first 10 of the 20 feeding ports, and the other subgroup of 9 could be used for another orthogonal beam.

(2) The second configuration shown in Figure 1 considers a similar linear array system of 20 antenna elements with 19 feeding ports (CORPS-BFN of one layer). In this configuration, the control of the 2 beams is realized by alternating input ports allowing that a group of 10 complex inputs could control the 
whole antenna array (20 antenna elements) for beam no. 1 , and the 9 inputs remaining can control 18 of 20 antenna elements for beam no. 2 .

The objective is to evaluate the behavior of the array factor generated by the two configurations of CORPSBFN for multibeam steerable linear arrays. Furthermore, the results are compared with the same configurations of CORPS-BFN under study but adding the Matrix Pencil Method to the antenna elements for a possible performance improvement.

The method of PSO was implemented to find a set of complex inputs to feed the configurations of CORPS-BFN shown in Figures 2 and 3 based in the objective function (9). This is in order to study the behavior of the array factor generated by both configurations (reference patterns). The evaluation of the array factor considers a steering range of $80^{\circ}$, with an angular step of $10^{\circ}$ for a linear array of 20 antenna elements with an initial uniform spacing of $0.5 \lambda$. We have set the parameters of PSO based on our previous experience in solving antenna problems $[2,10]$. After a trial and error procedure for parameter tuning, the parameters of the algorithm were set as follows: the PSO algorithm is executed using 200 particles (population size) through out 500 iterations to ensure a good sampling of the solution space. A global topology of PSO is used with time-varying inertial weight $(\omega)$ that varies from 0.9 to 0.4 through out the iterations, the acceleration constants $\left(c_{1}, c_{2}\right)$ are set to 2.0 and the maximum allowed velocity $\left(v_{d, \max }\right)$ is set as $\omega r$ where $r=\left(v_{d, \max }-v_{d, \min }\right)$ in $(7)[2,10-12]$.

In the case of the MPM, the error $\xi$ of the reconstructed or approximated pattern generated is defined as

$$
\xi=\frac{\int_{-1}^{1}\left|\overline{\mathrm{AF}}_{\mathrm{ref}}(u)-\overline{\mathrm{AF}}_{\mathrm{MPM}}(u)\right|^{2} d u}{\int_{-1}^{1}\left|\overline{\mathrm{AF}}_{\mathrm{ref}}(u)\right|^{2} d u}
$$

The metric in (15) assesses the degree of optimality of the redesigned array defining the error over the reconstructed array factor $\overline{\mathrm{AF}}_{\mathrm{MPM}}$ with respect to the reference $\overline{\mathrm{AF}}_{\text {ref }}$.

3.2. Simulation Results. To develop a comprehensive comparative analysis of the linear array system that just considers CORPS-BFN and the system with CORPS-BFN plus the Matrix Pencil Method, first we analyze the behavior of the array factor in the two configurations of CORPS-BFN (Figures 2 and 3 ).

Figures 4 and 5 show an example of the radiation pattern of a 2-beam system generated by the configuration 1 of CORPS-BFN. This particular example shows the beam no. 1 (controlling 10 inputs for 11 outputs in Figure 4) and beam no. 2 (controlling 9 inputs for 10 outputs in Figure 5) of the configuration 1 (Figure 2). This configuration was previously optimized by PSO showing the CORPS-BFN capabilities to handle SLL and for scanning the beam to a specific spatial locations with certain directivity. In Figure 4, the specific values obtained of side lobe level (SLL) and directivity $(D)$ are -25.40 and $9.72 \mathrm{~dB}$, respectively. In the same way, in Figure 6 the values are SLL $=-25.12 \mathrm{~dB}$ and $D=9.34 \mathrm{~dB}$.
Furthermore, this one-dimensional array factor obtained in each figure defines the reference for the compression process realized by the MPM. The array factor response of this additional method added to the configuration of CORPSBFN (reconstructed pattern) is also shown in Figures 4 and 5 and compared directly with respect to the response of CORPS-BFN (reference pattern). As can be seen in both figures, the CORPS-BFN plus the MPM almost exactly reproduce the desired pattern generated by the CORPS-BFN with $\xi=0.041$ at $100^{\circ}$ for beam no. 1 (Figure 4 ) and $\xi=0.012$ at $80^{\circ}$ for beam no. 2 (Figure 5) (for values of $\xi$ in all the steering range, see Table 1). In the reduction process of the MPM it is defined the number of samples of the reference array factor (sampling points). From Nyquist sampling theorem at least $(2 N-1)$ samples are suitable.

Similarly, Figure 6 shows the same comparison made in Figures 4 and 5 considering the configuration 2 of the CORPS-BFN for the beam no. 1. In this specific example the beam is controlled by 10 inputs ports for 20 outputs (by alternating inputs ports see Figure 3). Despite the whole array is controlled by the half of input ports, the array factor formed by CORPS-BFN (reference pattern) in this example shows high levels of side lobe level (SLL $\approx$ $-5 \mathrm{~dB})$ and directivity $(D=10.84 \mathrm{~dB})$. The approximation pattern generated by the CORPS-BFN plus the MPM in this configuration presents a value of $\xi=0.137$ at $70^{\circ}$ for beam no. 1 showing side lobes almost similar to the main lobe. This could be a disadvantage in some steering directions. However a complete analysis of configuration 2 is presented in Table 1.

Additionally, in Figure 7 is represented the singular value spectrum of the CORPS-BFN pattern samples for the configurations of CORPS-BFN shown in Figures 4 and 5. The singular values in the two cases decay rapidly with just few small singular values that can be discarded. Based on this, the reference pattern can be reconstructed with an smaller aperture (see Figures 8 and 9). Furthermore, in Table 2 the values of $\epsilon$ (from (12)) for each angle of steering for the configuration 1 are shown ( $\epsilon$ depends on how accurately the reconstructed pattern approximates the reference radiation pattern).

The comparison between the example of CORPS-BFN (reference pattern) and the CORPS-BFN plus the MPM (reconstructed pattern) in terms of amplitude and element location $(\lambda)$ is shown in Figures 8 and 9. As it can be seen in both figures, the MPM redistributes the magnitude of the current and the location of each antenna element taking advantage of the non-uniformity of the linear array. This generates a reduction of the antenna elements. Given that the compression for beam no. 1 (Figure 8) are 2 antenna elements and for beam no. 2 is just 1 antenna element (Figure 9) in a CORPS-BFN of one layer (our case study where $M=N-1$ ) implies that also reduces 2 input ports for beam no. 1 (i.e., the 10 inputs $\times 11$ outputs for beam no. 1 are reduced to 8 inputs $\times 9$ outputs to generate the beam) and 1 input port for beam no. 2 (i.e., the 9 inputs $\times 10$ outputs for beam no. 2 are reduced to 8 inputs $\times$ 9 outputs to generate the beam). If we consider the entire system (i.e., beam no. 1 and no. 2) there is a significant 


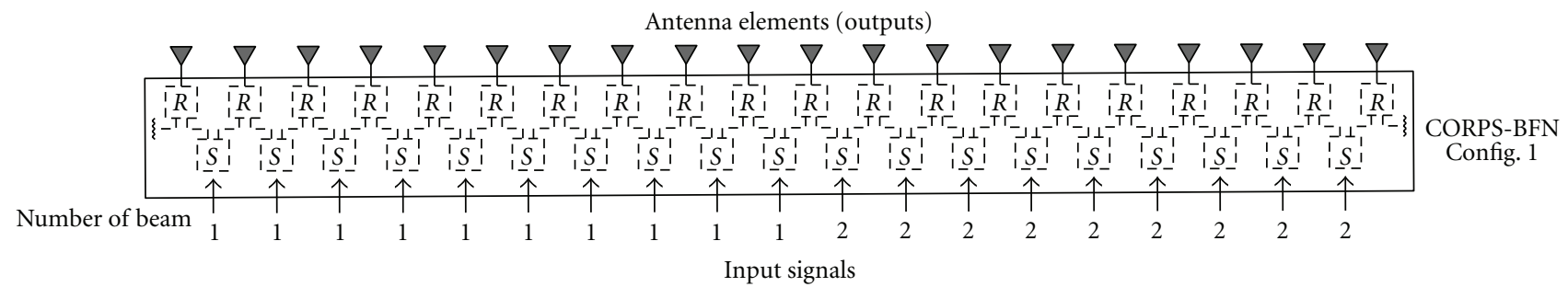

FIgURE 2: Configuration 1 of CORPS-BFN with 19 feeding ports and 20 antenna elements in a linear array considering 10 inputs $\times 11$ outputs for beam no. 1 and 9 inputs $\times 10$ outputs for beam no. 2 .



Figure 3: Configuration 2 of CORPS-BFN with 19 feeding ports and 20 antenna elements in a linear array considering 10 inputs $\times 20$ outputs for beam no. 1 and 9 inputs $\times 18$ outputs form beam no. 2 .

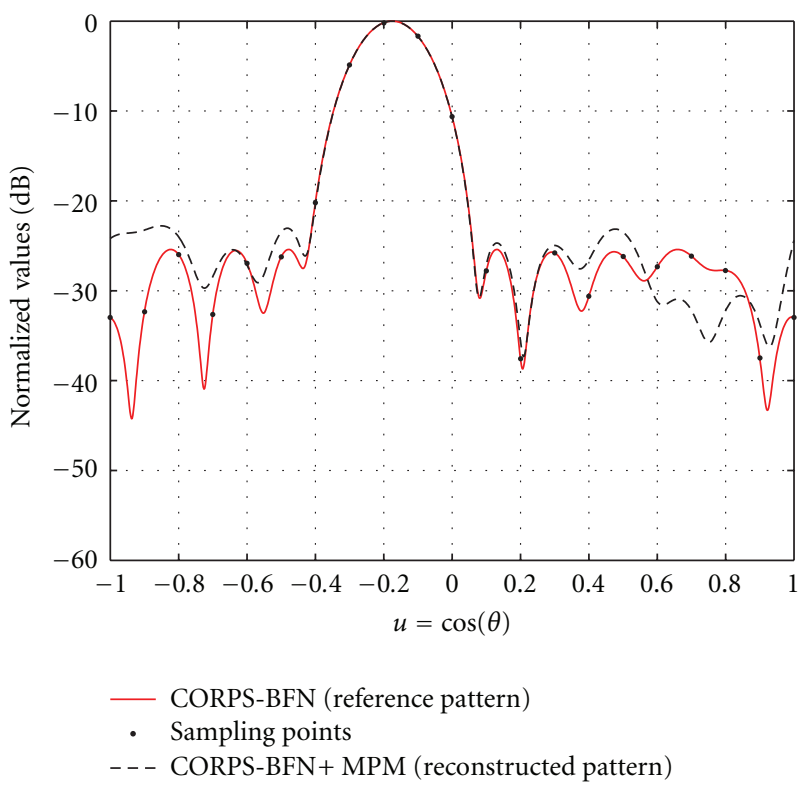

FIGURE 4: Comparison between the reference factor (CORPS-BFN) and the reconstructed factor (MPM + CORPS-BFN) obtained by the configuration 1 of the CORPS-BFN, showing an example of the beam no. 1 directed at $100^{\circ}$.

reduction of the whole CORPS-BFN system. Considering the complete configuration 1 , the 19 feeding ports, and 20 antenna elements are reduced to 16 feeding ports and 17 antenna elements considering 8 inputs for 9 outputs for beam no. 1 and 8 inputs for 9 outputs for beam no. 2 with a good approximation of the reference patterns.

To develop a more complete analysis of the two configurations under study, we analyzed the tradeoff between

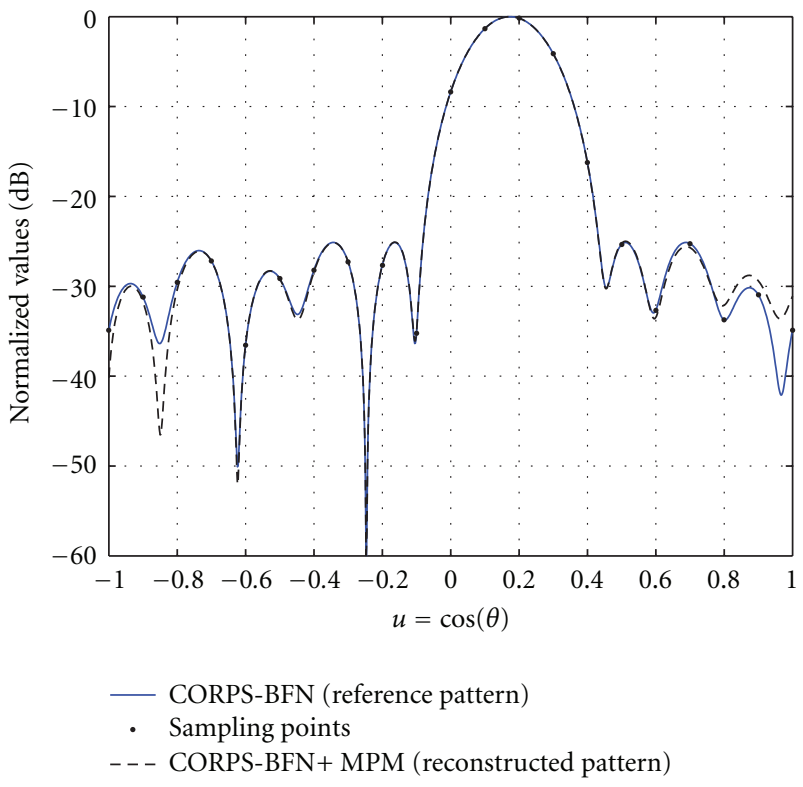

FIGURE 5: Comparison between the reference factor (CORPS-BFN) and the reconstructed factor (MPM + CORPS-BFN) obtained by the configuration 1 of the CORPS-BFN, showing an example of the beam no. 2 directed at $80^{\circ}$.

the steering angle (window of visibility) and the error of the reconstructed pattern (15) generated by the MPM. The Figures 10 and 11 show the error of the beam no. 1 (line with squares) and beam no. 2 (line with diamonds) for each configuration of CORPS-BFN (Figures 2 and 3) analyzed. The scanning range is considered between $50^{\circ} \leq \theta_{0} \leq$ $130^{\circ}$ and $70^{\circ} \leq \theta_{0} \leq 110^{\circ}$ with an angular step of $10^{\circ}$ for the configuration 1 (Figure 10) and 2 (Figure 11) of 
TABLE 1: Error $\xi$ for the antenna steering in each evaluated scheme.

\begin{tabular}{|c|c|c|c|c|}
\hline \multirow{3}{*}{ Angle (Deg) } & \multicolumn{4}{|c|}{ Error $\xi$} \\
\hline & \multicolumn{2}{|c|}{ Configuration 1} & \multicolumn{2}{|c|}{ Configuration 2} \\
\hline & Beam \#1 & Beam \#2 & Beam \#1 & Beam \#2 \\
\hline 50 & 0.4177 & 0.3612 & - & - \\
\hline 60 & 0.4741 & 0.4863 & - & - \\
\hline 70 & 0.0299 & 0.0770 & 0.1372 & 0.0973 \\
\hline 80 & 0.0136 & 0.0124 & 0.4412 & 0.4116 \\
\hline 90 & 0.0224 & 0.0039 & 0.1332 & 0.0302 \\
\hline 100 & 0.0411 & 0.0177 & 0.4771 & 0.4097 \\
\hline 110 & 0.0698 & 0.0524 & 0.1015 & 0.0973 \\
\hline 120 & 0.5200 & 0.5067 & - & - \\
\hline 130 & 0.5294 & 0.2546 & - & - \\
\hline
\end{tabular}

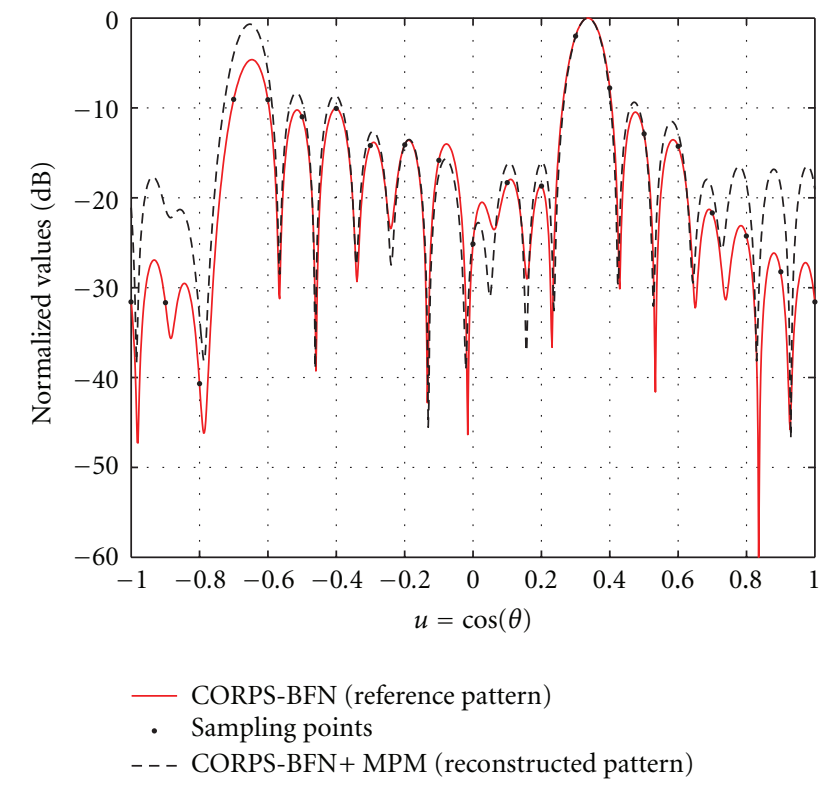

FIGURE 6: Comparison between the reference factor (CORPS-BFN) and the reconstructed factor (MPM + CORPS-BFN) obtained by the configuration 2 of the CORPS-BFN, showing an example of the beam no. 1 directed at $70^{\circ}$.

CORPS-BFN, respectively. The configuration 1 of CORPSBFN (Figure 10) shows a good performance in the scanning capability for the 2 beams analyzed with high values of error only at the ends of the steering range over the window of visibility $\left(50^{\circ}-130^{\circ}\right)$. For the case of the configuration 2 (Figure 11), both beams present a similar behavior with a small difference between them. In the configuration, there are two regions with high numerical values of error $\xi$ (values between 0.4 to 0.5 ). The first region has its peak at $80^{\circ}$ and the second at $100^{\circ}$ in a smaller window of visibility $\left(70^{\circ}-\right.$ $\left.110^{\circ}\right)$.

Although two specific cases to generate two scannable beams were presented, it is possible to define different configurations by varying the number of antenna elements
TABLE 2: Value of $\epsilon$ for each angle of steering.

\begin{tabular}{lcc}
\hline Angle (deg) & \multicolumn{2}{c}{ Value of $\epsilon$} \\
\hline 50 & Beam \#1 & Beam \#2 \\
60 & $10^{-8}$ & $10^{-7}$ \\
70 & $10^{-8}$ & $10^{-8}$ \\
80 & $10^{-7}$ & $10^{-8}$ \\
90 & $10^{-5}$ & $10^{-8}$ \\
100 & $10^{-5}$ & $10^{-5}$ \\
110 & $10^{-7}$ & $10^{-5}$ \\
120 & $10^{-7}$ & $10^{-8}$ \\
130 & $10^{-8}$ & $10^{-9}$ \\
\hline
\end{tabular}

and the number of input ports depending on the number of independent beams required. In general, it is important to note the significative simplification offered by the combination of CORPS-BFN with the compressive method of MPM. On one side the intrinsic technological advantages offered by CORPS-BFN itself (e.g. reuse of antenna elements between adjacent beams, reduction of control signal inputs, etc.). On the other hand, the MPM improvements added to the CORPS-BFN technology and demostrated in this paper based in the reduction of the aperture, taking advantage of the relocation of antenna elements (using the nonuniformity of the antenna array), and the redistribution of complex excitations. Thus, a reduction in the antenna array by the MPM generates a simplification in the CORPS-BFN (including all the associated circuitry, i.e., inputs ports, power combiners, and splitters). The above improvements offer a substantial simplification of the feeding network for linear arrays and for the system in general.

In this way, the lower complexity of an entire antenna system (i.e., the antenna array and the beam-forming network sub system) obtained by the CORPS-BFN plus the Matrix Pencil Method makes the system a good candidate for a great number of applications. Although our examples of CORPS-BFN plus the MPM for linear arrays are based on simulations, depending on the design requirements and 


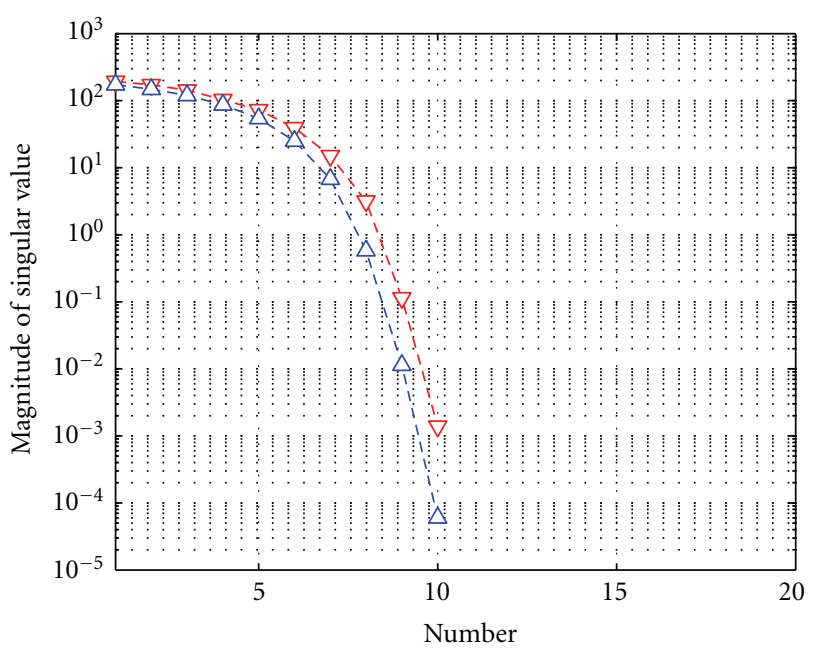

$-\nabla^{-}$Configuration 1 beam no. 1

$-\triangle$ - Configuration 1 beam no. 2

FIgURE 7: Singular value spectrum of the CORPS-BFN configuration 1, for the example based in Figures 4 and 5.



Figure 8: Amplitude distribution and antenna element location for the reference factor (CORPS-BFN) and the reconstructed factor $(\mathrm{MPM}+\mathrm{CORPS}-\mathrm{BFN})$ for the beam no. 1 shown in Figure 4.

possible application, more suitable configurations can be set and then be implemented.

\section{Conclusion}

The design of a simplified feeding network based on CORPS technology plus the non-iterative MPM for scannable multibeam antenna arrays in a linear geometry has been presented. The design approach involved the study of the behavior of

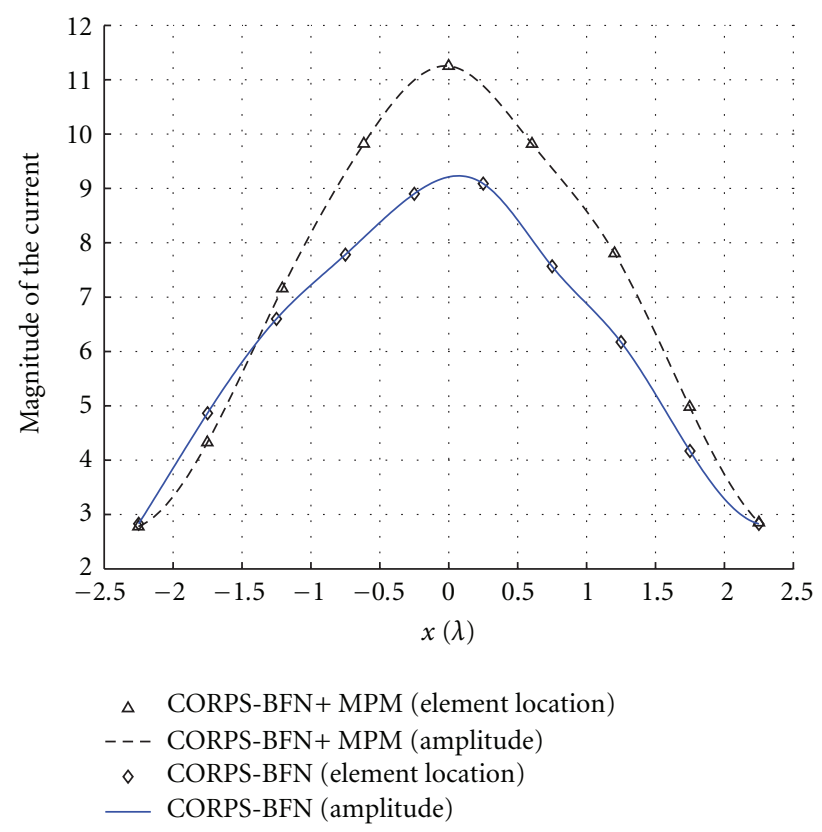

Figure 9: Amplitude distribution and antenna element location for the reference factor (CORPS-BFN) and the reconstructed factor $(\mathrm{MPM}+\mathrm{CORPS}-\mathrm{BFN})$ for the beam no. 2 shown in Figure 5.

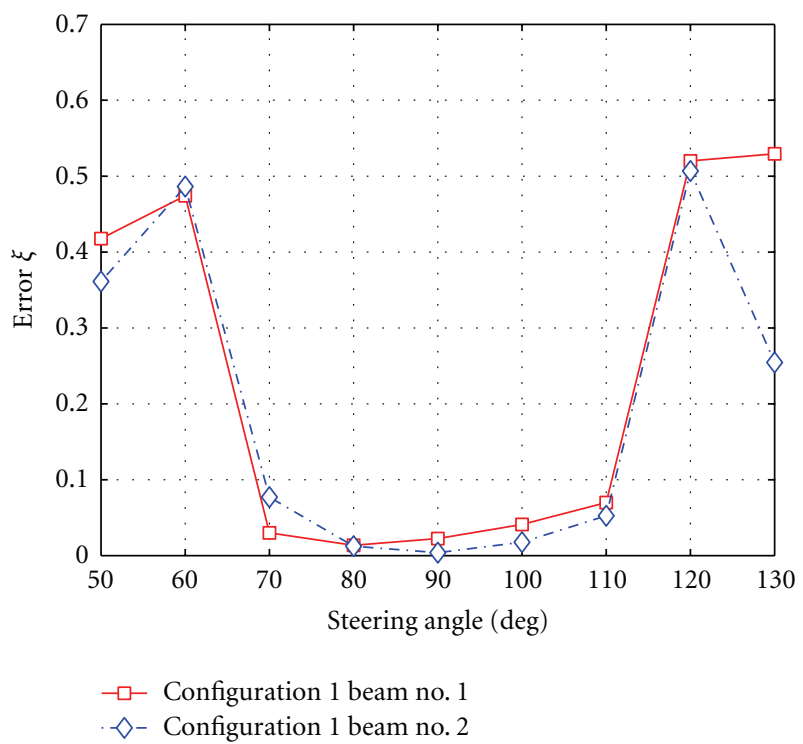

FIGURE 10: Behavior of the error $\xi$ with respect to the direction of maximum gain for the configuration 1 of CORPS-BFN.

the array factor generated by the combination of CORPSBFN plus the MPM for a multi-beam steerable linear array. Simulation results reveal that the design of CORPSBFN with added matrix pencil method could generate independent and scannable beams in linear arrays exploiting the non-uniformity of the antenna array with a significant simplification of the entire antenna system. Furthermore, the CORPS-BFN configurations showed the advantages and limitations of applying the non-iterative MPM in linear 


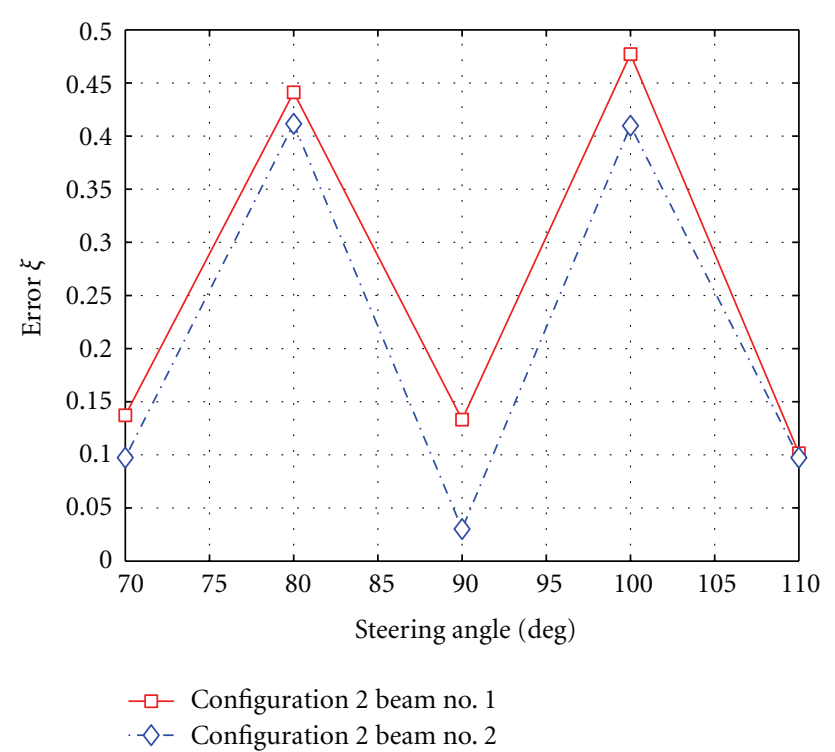

FIgURE 11: Behavior of the error $\xi$ with respect to the direction of maximum gain for the configuration 2 of CORPS-BFN.

arrays and in applications that involve multiple independent beams and beam steering capabilities.

\section{Acknowledgment}

This work was supported by the Mexican National Science and Technology Council, CONACYT, under Grant 127919.

\section{References}

[1] D. Betancourt and C. del Río Bocio, "A novel methodology to feed phased array antennas," IEEE Transactions on Antennas and Propagation, vol. 55, no. 9, pp. 2489-2494, 2007.

[2] A. Arce, D. H. Covarrubias, and M. A. Panduro, "Design of a multiple-beam forming network using CORPS optimized for cellular systems," AEU-International Journal of Electronics and Communications, vol. 66, no. 5, pp. 349-356, 2012.

[3] A. Arce, D. H. Covarrubias, M. A. Panduro, and L. A. Garza, "A new multiple-beam forming network design approach for a planar antenna array using CORPS," Journal of Electromagnetic Waves and Applications, vol. 26, pp. 294-306, 2012.

[4] N. Ferrando and N. J. G. Fonseca, "Investigations on the efficiency of array fed coherently radiating periodic structure beam forming networks," IEEE Transactions on Antennas and Propagation, vol. 59, no. 2, pp. 493-502, 2011.

[5] M. A. Panduro and C. del Rio-Bocio, "Simplifying the feeding network for multi-beam circular antenna arrays by using corps," Progress in Electromagnetics Research Letters, vol. 21, pp. 119-128, 2011.

[6] Y. Liu, Z. Nie, and Q. H. Liu, "Reducing the number of elements in a linear antenna array by the matrix pencil method," IEEE Transactions on Antennas and Propagation, vol. 56, no. 9, pp. 2955-2962, 2008.

[7] Y. Hua and T. K. Sarkar, "Matrix pencil method for estimating parameters of exponentially damped/undamped sinusoids in noise," IEEE Transactions on Acoustics, Speech, and Signal Processing, vol. 38, no. 5, pp. 814-824, 1990.
[8] C. A. Balanis, Antenna Theory: Analysis and Design, WileyInterscience, New Jersey, NJ, UA, 3rd edition, 2005.

[9] R. Eberhart, Y. Shi, and J. Kennedy, Swarm Intelligence, Morgan Kaufmann, San Francisco, Calif, USA, 1st edition, 2005.

[10] A. Arce, D. H. Covarrubias, and M. A. Panduro, "Performance evaluation of population based optimizers for the synthesis of linear antenna arrays," in Proceedings of the 6th IASTED International Conference on Antennas, Radar, and Wave Propagation, vol. 649, pp. 103-108, 2011.

[11] R. C. Eberhart and Y. Shi, "Particle swarm optimization: developments, applications and resources," in Proceedings of the Congress on Evolutionary Computation, vol. 1, pp. 81-86, May 2001.

[12] N. Jin and Y. Rahmat-Samii, "Advances in particle swarm optimization for antenna designs: real-number, binary, singleobjective and multiobjective implementations," IEEE Transactions on Antennas and Propagation, vol. 55, no. 3, pp. 556-567, 2007. 

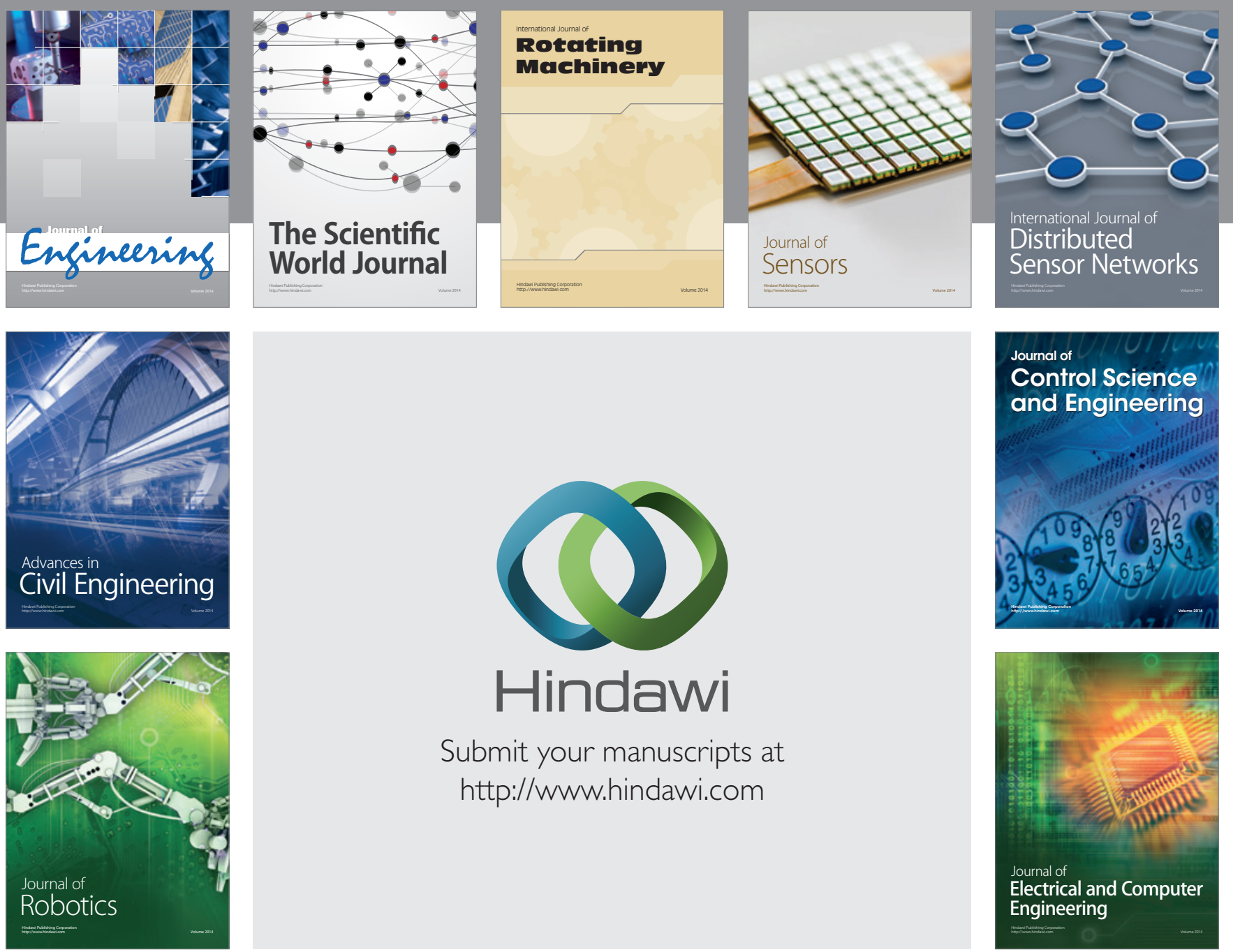

Submit your manuscripts at

http://www.hindawi.com
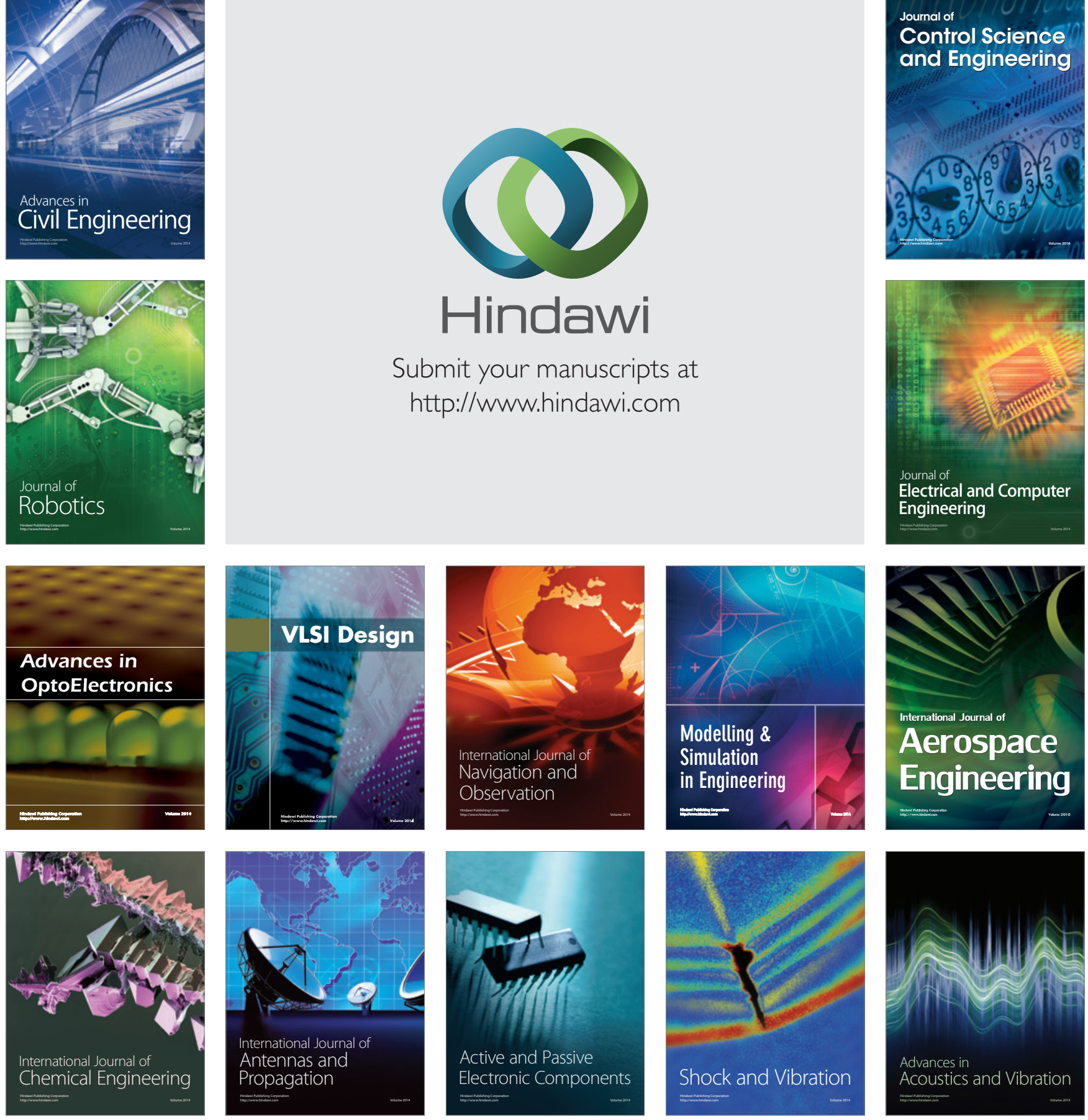